\title{
Unique range sets in positive characteristic
}

\author{
by \\ AbDelbaki BoutabaA (Clermont-Ferrand), \\ William Cherry (Denton, TX) and \\ Alain Escassut (Clermont-Ferrand)
}

1. Introduction. In the course of studying the factorization of meromorphic functions (on the complex plane), Gross [13] introduced the concept of a unique range set, which we now define. Let $f$ be a function for which it makes sense to talk about the "multiplicity" with which it takes on a value. For example, $f$ could be a non-constant polynomial or an entire function. Let $S$ be a set in the range of $f$. Then, define

$$
E(f, S)=\bigcup_{a \in S}\{(z, m): f(z)=a \text { with multiplicity } m\} .
$$

Here $z$ runs over the domain of $f$ and $m$ is any positive integer. Two functions $f$ and $g$ of the same type are said to share $S$ (with multiplicity) if $E(f, S)=E(g, S)$. A set $S$ is called a unique range set (counting multiplicity) for a family of functions $\mathcal{F}$, if whenever one has $E(f, S)=E(g, S)$ for $f, g \in \mathcal{F}$, then one must have $f \equiv g$.

Providing examples of unique range sets for (non-constant) complex entire functions has been the subject of a number of recent papers. Nevanlinna theory in one form or another has been the main tool used to construct those examples. See [16] for a recent survey.

Boutabaa, Escassut, and Haddad [7] were the first to study unique range sets for non-Archimedean entire functions (in characteristic zero) and as part of their study, they found that if one restricts oneself to the study of polynomials, then there is a nice geometric characterization for finite unique range sets. Before stating their theorem, we recall that a set is called affinely rigid if the only affine transformation preserving the set is the identity.

Theorem 1.1 (Boutabaa, Escassut, and Haddad [7]). Let $K$ be a field of characteristic zero. Let $\mathcal{F}$ be the family of non-constant polynomials with

2000 Mathematics Subject Classification: Primary 12E05; Secondary 11C08, 11S80, $30 \mathrm{D} 35$. 
coefficients in $K$. Then a finite set $S$ in $K$ is a unique range set for $\mathcal{F}$ if and only if $S$ is affinely rigid.

Cherry and Yang [9] extended this theorem to the case of non-constant non-Archimedean entire functions in one variable over a field of characteristic zero, complete with respect to a non-Archimedean absolute value.

In the following, $K$ will always be a field complete with respect to a nonArchimedean absolute value. "Unique range set" will always mean unique range set counting multiplicity for the family $\mathcal{A}^{*}(K)$ of non-constant nonArchimedean entire functions on $K$. We can consider polynomials over any field to be a special case. Namely, let $K$ be any field. The "trivial" absolute value ||$_{0}$ on $K$ is defined as follows. For $x \in K$ with $x \neq 0$, we have $|x|_{0}=1$, and $|0|_{0}=0$. This is clearly a non-Archimedean absolute value on $K$, and $K$ is clearly complete with respect to this absolute value. Moreover, the one-variable non-Archimedean entire functions on $K$ are simply the one-variable polynomials with coefficients in $K$. Hence, whenever we state something for the family of non-Archimedean entire functions in one variable, the statement remains true for polynomials.

Voloch [20] gave a purely "algebro-geometric" proof of Theorem 1.1 that makes it clear that the same result holds in positive characteristic for sets with cardinality prime to $n$. Namely,

TheOREM 1.2 (Voloch [20]). Let $K$ have characteristic $p \geq 0$ and be complete with respect to a non-Archimedean absolute value. Let $\mathcal{A}^{*}(K)$ be the family of non-constant non-Archimedean entire functions on $K$. Let $S$ be a set of finite cardinality $n$, assumed prime to $p$ if $p>0$. Then $S$ is a unique range set for $\mathcal{A}^{*}(K)$ if and only if $S$ is affinely rigid.

Voloch's argument is reproduced in an appendix to the recent work of Wang [21], so we do not discuss this in more detail here. The purpose of our work is to investigate what can happen when the characteristic $p$ divides the cardinality of a set.

In [9], Cherry and Yang gave an example of a set of three elements which was affinely rigid, but which was not a unique range set in characteristic three. Voloch [20] remarked that his proof of Theorem 1.2 also shows there is no unique range set of cardinality three in characteristic three. In Section 2 we provide a proof of the non-existence of three-element unique range sets in characteristic three and we show that for every prime power $q=p^{n} \geq 3$, there exist affinely rigid sets of cardinality $q$ which are not unique range sets in characteristic $p$. Our examples are constructed in such a way that one sees clearly how the Frobenius morphism is exploited to create these examples. One might expect that a set which is affinely rigid and not invariant under some process involving the Frobenius morphism should be a unique range set. Unfortunately, we are not able to formulate a precise conjecture in 
this direction, much less prove such a characterization. We hope that the examples given in this paper will stimulate further research in this direction.

Because there are no affinely rigid sets of cardinality two, there are also no unique range sets of cardinality two in characteristic two (or in any characteristic). That raised the question of whether there could be unique range sets of cardinality $p$ in characteristic $p$. In Section 3 , we give examples of unique range sets of cardinality $n$ in all characteristics for all $n \geq 4$. NonArchimedean Nevanlinna theory, which was recently extended to positive characteristic by Boutabaa and Escassut in [6], still seems to be the most useful technique available for constructing examples of unique range sets. However, for small cardinality, it seems to be easier to use algebro-geometric methods. Our algebro-geometric techniques remain rather ad-hoc. A more thorough understanding of the possible rational components of plane curves of the form $P(X)-c P(Y)=0$ in positive characteristic, where $P$ is a polynomial and $c$ is a constant, is probably necessary before a complete geometric characterization of unique range sets in positive characteristic can be given.

We leave open the problem of whether there exist affinely rigid finite sets of cardinality $n$ which are not unique range sets, and where $n$ is a multiple, but not a pure power, of the characteristic.

We conclude our introduction by mentioning the work of Ostrovskii, Pakovitch, and Zaidenberg [17], where they consider the more difficult question of unique range sets ignoring multiplicity. That is, they consider sets $S$ such that $f^{-1}(S)=g^{-1}(S)$ implies $f \equiv g$. Ostrovskii, Pakovitch, and Zaidenberg prove that a finite set of complex numbers is a unique range set ignoring multiplicity for the set of complex polynomials of fixed degree $d \geq 1$ on the complex plane if and only if $S$ is affinely rigid. The methods of proof in [17] are metric based, and therefore it would be very interesting to find an algebraic proof that generalizes their work to positive characteristic, even in the case where the cardinality of $S$ is prime to $p$.

Acknowledgements. Financial support for the second author to spend a pleasant month in Clermont-Ferrand was generously provided by a Junior Faculty Research Fellowship from the University of North Texas and by l'Université Blaise Pascal (Clermont-Ferrand).

2. Affinely rigid non-unique range sets in positive characteristic. Fundamental to everything we do in the rest of this paper is the following proposition.

Proposition 2.1. Let $K$ be an algebraically closed field, complete with respect to a non-Archimedean valuation. Let $S$ be a finite set in $K$, and let $P(x)=\prod_{s \in S}(x-s)$ be the smallest degree monic polynomial with coefficients in $K$, whose set of roots is $S$. Then two functions $f$ and $g$ in $\mathcal{A}^{*}(K)$ share 
$S$ counting multiplicity if and only if there exists a non-zero constant $c \in K$ such that $P(f)=c P(g)$.

Proof. If $P(f)=c P(g)$, then $P(f)=0$ if and only if $P(g)=0$, and both functions vanish with the same multiplicity. Thus, $f$ and $g$ clearly share $S$ (counting multiplicity). Alternatively, if $f$ and $g$ share $S$ counting multiplicity, then $P(f) / P(g)$ is a non-Archimedean analytic function on $K$ without zeros, hence constant. This last fact is classical following easily from the theory of valuation (or Newton) polygons. It also follows from Theorem 4.3.

We will use Proposition 2.1 continually throughout this paper and will not always refer to it explicitly.

In this section we exploit the Frobenius morphism to construct examples of sets which are affinely rigid, but not unique range sets in positive characteristic.

We remark that if a set is not a unique range set for the family of non-constant polynomials, it is also not a unique range set for the family of non-constant non-Archimedean entire functions, so in this section it suffices to consider polynomials.

EXAMPLE 2.2. Let $K$ be an algebraically closed field of characteristic $p>0$. Let $n$ be an integer such that $q=p^{n} \geq 3$. Let

$$
P(X)=X^{q}+(X-1)^{q-1} .
$$

Let $f(z)=z^{q-1}$ and $g(z)=(z-1)^{q-1}$. Then

$$
P(f(z))=P(g(z)) \text {. }
$$

Moreover $P$ has $q$ distinct zeros and the set $S$ of zeros of $P$ gives an example of an affinely rigid set with $q$ points that is not a unique range set for nonconstant polynomials with coefficients in $K$.

Proof. We first show $P(f(z))=P(g(z))$. As it turns out, it is easier to show

$$
P(f(z))\left(z^{q}-z\right)=P(g(z))\left(z^{q}-z\right),
$$

which still allows us to conclude $P(f(z))=P(g(z))$. Indeed,

$$
\begin{aligned}
P(f(z))\left(z^{q}-z\right) & =\left[z^{q(q-1)}+\left(z^{q-1}-1\right)^{q-1}\right]\left(z^{q}-z\right) \\
& =z^{q^{2}}-z^{q(q-1)+1}+z\left(z^{q-1}-1\right)^{q} \\
& =z^{q^{2}}-z^{q(q-1)+1}+z^{q(q-1)+1}-z=z^{q^{2}}-z .
\end{aligned}
$$

Similarly,

$P(g(z))\left(z^{q}-z\right)=P(g(z))\left((z-1)^{q}-(z-1)\right)=(z-1)^{q^{2}}-(z-1)=z^{q^{2}}-z$.

Thus, $P(f(z))=P(g(z))$ as claimed. 
Note that

$$
P(X)=\frac{X^{q+1}-1}{X-1}
$$

and hence the zeros of $P$ are precisely the $q+1$-st roots of unity, except for 1 . It remains to show that $S$ is affinely rigid. Note that

$$
\sum_{s \in S} s=-1
$$

Suppose $S$ is invariant by an affine transformation $\sigma(z)=a z+b$. Then we would also have

$$
-1=\sum_{s \in S} \sigma(s)=q b+a \sum_{s \in S} s=-a .
$$

Note $q b=0$, since we are in characteristic $p$. Hence $a=1$. It remains to show that $b=0$, and note that thus far we have not used the hypothesis $q \geq 3$.

For every $s$ in $S$, we know $s+b$ is also in $S$ and so $(s+b)^{q+1}=1$. Thus,

$$
1=(s+b)^{q+1}=(s+b)\left(s^{q}+b^{q}\right)=s^{q+1}+s^{q} b+s b^{q}+b^{q+1} .
$$

Of course $s^{q+1}=1$, so $b\left(s^{q}+s b^{q-1}+b^{q}\right)=0$, and if $b \neq 0$, we conclude that

$$
b^{q}+s b^{q-1}+s^{q}=0 .
$$

Adding this equation over all $s \in S$, and using the fact that $\sum_{s \in S} s=-1$, we get

$$
0=q b^{q}+b^{q-1} \sum_{s \in S} s+\sum_{s \in S} s^{q}=-b^{q-1}+\left(\sum_{s \in S} s\right)^{q}=-b^{q-1}+(-1)^{q} .
$$

Thus, $b^{q-1}=(-1)^{q}=-1$. Hence, we can simplify equation (1) to

$$
b=s^{q}-s .
$$

We now consider two cases:

Case $p \geq 3$. Add equation (2) over all $s$ in $S$ except for -1 to conclude that

and thus $b=0$.

$$
(q-1) b=\sum_{s \neq-1} s^{q}-\sum_{s \neq-1} s=\left(\sum_{s \neq-1} s\right)^{q}-\sum_{s \neq-1} s=0-0=0
$$

Case $p=2$. Write $S=\left\{\zeta, \zeta^{2}, \ldots, \zeta^{2^{n}}\right\}$. Then equation (2) becomes

$$
b=\zeta^{2^{n}+1-j}+\zeta^{j} \quad \text { for all } j
$$

noting that $\zeta^{2^{n} j}=\zeta^{2^{n}+1-j}$ since $\zeta^{2^{n}+1}=1$. Adding these equations for $j=1, \ldots, 2^{n-1}$, we get

$$
2^{n-1} b=\sum_{j=1}^{2^{n}} \zeta^{j}=1 .
$$

This implies $n=b=1$, but since $n \geq 2$ by hypothesis, we conclude that $b=0$. 
As first remarked by Voloch [20], Example 2.2 is typical for three-point sets in characteristic three. Namely, there are no unique range sets of cardinality three. We first give Voloch's geometric proof, and then we give another proof by "brute force," which has the advantage of giving concrete examples.

TheOREM 2.3. Let $K$ be a field of characteristic three. Then there are no three-element unique range sets for the family of non-constant polynomials with coefficients in $K$.

Voloch's proof. Let $S=\left\{s_{1}, s_{2}, s_{3}\right\}$ and $P(x)=\left(x-s_{1}\right)\left(x-s_{2}\right)\left(x-s_{3}\right)$. Then

$$
G(x, y)=\frac{P(x)-P(y)}{x-y}
$$

defines a quadric curve in $\mathbb{P}^{2}$. Because $P(x)-P(y)$ has only a single point at infinity in characteristic 3, the same is true of the quadric defined by $G$. Hence, $G$ is parameterized by quadratic polynomials. This results in two different quadratic polynomials $f$ and $g$ such that $P(f)=P(g)$, which of course means $f$ and $g$ share $S$.

Brute force proof. Again, let $S=\left\{s_{1}, s_{2}, s_{3}\right\}$, and let

$$
P(x)=\left(x-s_{1}\right)\left(x-s_{2}\right)\left(x-s_{3}\right)=x^{3}+a_{2} x^{2}+a_{1} x+a_{0} .
$$

We consider two cases.

Case $a_{2}=0$. In this case $a_{1} \neq 0$, or otherwise, $P$ does not have distinct roots (in characteristic three). Choose an element $b$ in $K$ such that $b^{2}+a_{1}$ $=0$. Then

$$
P(z+b)=z^{3}+b^{3}+a_{1}(z+b)+a_{0}=P(z)+b\left(b^{2}+a_{1}\right)=P(z) .
$$

Hence, $S$ is not a unique range set.

Case $a_{2} \neq 0$. In this case, replacing $x$ by $x+a_{1} / a_{2}$ eliminates the linear term from $P$. Transforming $S$ by an affine transformation does not change whether or not it is a unique range set, so we may assume, without loss of generality that $a_{1}=0$. In this case, let

$$
f(z)=a_{2}\left(z^{2}-1\right) \quad \text { and } \quad g(z)=a_{2}\left(z^{2}+z\right) .
$$

Then

$$
P(f(z))=a_{2}^{3}\left(z^{6}+z^{4}+z^{2}\right)+a_{0}=P(g(z)),
$$

and again, $S$ is not a unique range set.

We conclude this section by remarking that it would be interesting to construct examples similar to Example 2.2 for multiples of $p$ which are not pure powers of $p$, or to prove that no such example exists. 
3. Examples of unique range sets. As we said in the introduction, finite unique range sets whose cardinality is prime to the characteristic have been completely characterized. We saw in the last section that the same characterization does not hold for sets with cardinality a power of the characteristic and that no unique range sets of cardinality three exist in characteristic three. In this section we give examples of unique range sets of cardinality $\geq 4$ in every characteristic.

Throughout this section, $K$ will be an algebraically closed field complete with respect to a non-Archimedean absolute value and $\mathcal{A}^{*}(K)$ will be the non-constant non-Archimedean entire functions on $K$.

First we recall some notation. We let $(m, n)$ denote the greatest common divisor of two integers $m$ and $n$. For a prime number $p$, we let $|n|_{p}$ denote the $p$-adic absolute value of an integer $n$. That is, if $p^{e}$ is the largest power of $p$ dividing $n$, then we let $|n|_{p}=p^{-e}$. Our main theorem is then the following:

Theorem 3.1. Let $K$ have characteristic $p \geq 0$. Let

$$
P(x)=x^{n}-a x^{m}+1
$$

with $n>m$. Assume that $a \neq 0$ in $K$ is such that

$$
m^{m}(n-m)^{n-m} a^{n} \neq n^{n}
$$

and

$$
m^{m}(n-m)^{n-m} a^{n} \neq n^{n}(1-\zeta)^{p^{d}(n-m)}
$$

for every integer $d \geq 0$, and for every $\zeta \in K$ such that

$$
\zeta^{n-m}=(-1)^{n-m} .
$$

Assume further that $m$ and $n$ satisfy EITHER conditions (A1) through (A3) OR (B1) through (B2) below.

(A1) $n>m>1$

(A2) $|(n, m)|_{p}=1$,

(A3) $n|n|_{p}$ does not divide $m$,

(B1) $n-2 \geq m \geq 5$,

(B2) $(n, m)=1$.

Then the set $S$ of zeros of $P$ in $K$ has $n$ elements and is a unique range set for $\mathcal{A}^{*}(K)$.

REMARK. For $d$ sufficiently large,

$$
(1-\zeta)^{p^{d}}=1-\zeta
$$

and so the condition that

$$
m^{m}(n-m)^{n-m} a^{n} \neq n^{n}(1-\zeta)^{p^{d}(n-m)}
$$

hold for all $d \geq 0$ simply means $a$ needs to be chosen so that it does not satisfy a finite number of algebraic equations. Hence, over an infinite field, 
and in particular over algebraically closed fields, one can always find such an $a$.

We postpone the proof of Theorem 3.1 until the next section in order to state some corollaries of the main theorem that give examples of unique range sets of all cardinalities $\geq 6$ in all characteristics.

Corollary 3.2. Let $K$ be as in Theorem 3.1 and let

$$
P(x)=x^{n}-a x^{n-1}+1 .
$$

Assume that $n>2$ and not a power of $p$, the characteristic of $K$. Assume that $a \neq 0$ is such that

$$
(n-1)^{n-1} a^{n} \neq n^{n} \quad \text { and } \quad(n-1)^{n-1} a^{n} \neq 2 n^{n} .
$$

Then $S$, the set of zeros of $P$, has $n$ elements and is a unique range set for $\mathcal{A}^{*}(K)$.

REMARK. If the characteristic of $K$ is 2 and $n$ is even, then the condition $(n-1)^{n-1} a^{n} \neq 2 n^{n}$ is always satisfied for $a \neq 0$. If $n$ is odd, then this condition is never satisfied and the corollary does not apply. But in this case, Theorem 1.2 does apply.

Proof. Apply Theorem 3.1 with $n=n$ and $m=n-1$. Clearly $n|n|_{p}$ divides $n$ and is $>1$, since $n$ is not a power of $p$. Thus, since $n$ and $n-1$ are relatively prime, conditions (A1) through (A3) of the theorem are satisfied. Since $n-m=1$, the conditions on $a$ become

$$
(n-1)^{n-1} a^{n} \neq n^{n} \quad \text { and } \quad(n-1)^{n-1} a^{n} \neq n^{n} 2^{p^{d}(n-m)}
$$

for all integers $d \geq 0$. But, since $2^{p^{d}}=2$ for all $d \geq 0$, we can forget about the $p^{d}$ in the exponent.

Corollary 3.3. Let $K$ be as in Theorem 3.1 with characteristic $p \geq 3$. Let $n=p^{r} \geq 7$ for $r$ a positive integer, and let

$$
P(x)=x^{n}-a x^{n-2}+1 .
$$

Assume that $a \neq 0$ is such that

$$
4(n-2)^{n-2} a^{n} \neq n^{n} \quad \text { and } \quad(n-2)^{n-2} a^{n} \neq n^{n} .
$$

Then $S$, the set of zeros of $P$, has $n$ elements and is a unique range set for $\mathcal{A}^{*}(K)$.

Proof. Conditions (B1) and (B2) are satisfied.

Corollary 3.4. Let $K$ be as in Theorem 3.1 with characteristic 2. Let $n=2^{r} \geq 8$ for $r$ a positive integer, and let

$$
P(x)=x^{n}-a x^{n-3}+1 .
$$

Assume $a \neq 0$. Then $S$, the set of zeros of $P$, has $n$ elements and is a unique range set for $\mathcal{A}^{*}(K)$. 
Proof. Again, conditions (B1) and (B2) are satisfied, and the conditions on $a$ in the theorem are trivial in this case.

Neither Theorem 1.2 nor Theorem 3.1 covers the case of four-element sets in characteristic two or five-element sets in characteristic five. We now give specific examples in each of these two cases. Our method here is algebrogeometric. Namely, consider a monic polynomial $P(x)$ whose zeros are our set $S$ and distinct, and we consider the algebraic curves defined by

$$
F_{c}(x, y)=P(x)-c P(y)=0
$$

for $c \neq 0$. Then, if $f$ and $g$ are two non-Archimedean analytic functions that share $S$, then by Proposition 2.1, $f$ and $g$ must be solutions to the equation $F_{c}=0$ for some non-zero constant $c$. By Berkovich's non-Archimedean Picard theorem [3], this equation will have no solutions in non-constant non-Archimedean analytic functions provided $F_{c}$ has no rational components. Of course when $c=1, F_{1}$ will have the rational component $x-y=0$, corresponding to $f=g$. But, if there are no other rational components, then $S$ will be a unique range set. Thus, we simply choose polynomials $P(x)$ so that the curves $F_{c}$ have singularities which are easy to analyze (which is somewhat easier said than done).

In addition to the Berkovich theorem mentioned above, we use some basic facts about the geometry of plane curves. These are the following. A non-singular plane curve of degree $d$ has genus $g$ given by

$$
g=\frac{(d-1)(d-2)}{2}
$$

If a plane curve is cut out by a homogeneous form $F(X, Y, Z)$ the singular points of $F$ are given by the simultaneous vanishing of $F$ and its three first partial derivatives. A singular point $s$ is called an ordinary double point if we can find local coordinates $u$ and $v$ around $s$ such that

$$
F=u v+\text { higher order terms. }
$$

We also need the fact that an irreducible plane curve of degree $d$ with a single ordinary double point singularity has (geometric) genus

$$
g=\frac{(d-1)(d-2)}{2}-1 .
$$

In general, each ordinary double point drops the genus by one. For background on the geometry of plane curves, see for instance [12].

EXAmPle 3.5. Let $K$ have characteristic 2. Let $P(x)=x^{4}+x^{3}+x$. Then $S=\{z \in K: P(z)=0\}$ contains 4 points and is a unique range set for $\mathcal{A}^{*}(K)$.

Proof. We first show that $S$ has four elements. Factoring $P$, we see

$$
P(x)=x\left(x^{3}+x^{2}+1\right) .
$$


Because $x^{3}+x^{2}+1$ is irreducible over the field of two elements, it has three distinct roots in $K$, none of which are zero. Hence, $S$ has four elements.

To check that $S$ is a unique range set, and recalling that we are working in characteristic 2 , it suffices to check that the homogeneous form

$$
F_{c}(X, Y, Z)=X^{4}+X^{3} Z+X Z^{3}+c\left(Y^{4}+Y^{3} Z+Y Z^{3}\right)
$$

has no rational components when $c \neq 0,1$ and only has the linear rational component $X+Y$ when $c=1$.

We first consider the case $c=1$. Then

$$
\begin{aligned}
F_{1}(X, Y, Z) & =X^{4}+Y^{4}+X^{3} Z+Y^{3} Z+X Z^{3}+Y Z^{3} \\
& =(X+Y)^{4}+(X+Y)\left(X^{2}+X Y+Y^{2}\right) Z+(X+Y) Z^{3} \\
& =(X+Y)\left[(X+Y)^{3}+\left(X^{2}+X Y+Y^{2}\right) Z+Z^{3}\right] .
\end{aligned}
$$

We consider the form

$$
G(X, Y, Z)=(X+Y)^{3}+\left(X^{2}+X Y+Y^{2}\right) Z+Z^{3} .
$$

The partial derivatives are

$$
\begin{aligned}
& \frac{\partial G}{\partial X}=(X+Y)^{2}+Y Z, \\
& \frac{\partial G}{\partial Y}=(X+Y)^{2}+X Z, \\
& \frac{\partial G}{\partial Z}=X^{2}+X Y+Y^{2}+Z^{2} .
\end{aligned}
$$

Singular points are determined by the simultaneous vanishing of all three partials. For the first two partials to vanish, we must have $X Z=Y Z$, so either $Z=0$ or $X=Y$. But, if $Z=0$, we also need $X+Y=0$, and we again have $X=Y$. But, in this case, $Y Z=X Z=0$. If $Z=0$, for the third partial to vanish, we need $X Y=0$, and so all three variables would need to vanish, which is not allowed. If $X=Y=0$, but $Z \neq 0$, then $G$ does not vanish, and so the curve defined by $G$ is non-singular. Since $G$ has degree three, it has genus 1 , and we are done in the case $c=1$.

In the case $c \neq 0,1$, we have the following three partial derivatives for $F_{c}$ :

$$
\begin{aligned}
& \frac{\partial F_{c}}{\partial X}=Z\left(X^{2}+Z^{2}\right), \\
& \frac{\partial F_{c}}{\partial Y}=c Z\left(Y^{2}+Z^{2}\right), \\
& \frac{\partial F_{c}}{\partial Z}=X\left(X^{2}+Z^{2}\right)+c Y\left(Y^{2}+Z^{2}\right) .
\end{aligned}
$$

If $Z=0$, then the first two partials vanish. For the third partial to also vanish along with $F_{c}$, we need

$$
X^{3}+c Y^{3}=0 \quad \text { and } \quad X^{4}+c Y^{4}=0 .
$$


Let $b$ be the element of $K$ such that $b^{4}=c$. Then $X=b Y$. Thus,

$$
(b Y)^{3}+c Y^{3}=\left(b^{3}+c\right) Y^{3}=0 .
$$

If $Y=0$, then so does $X$, and since we are in the case of $Z=0$, this is not allowed. Hence, $b^{3}+c=0$. Multiplying by $b$, we get

$$
0=b^{4}+c b=c+c b=c(1+b),
$$

so either $c=0$ or $b=1$. The case $c=0$ is not allowed, and if $b=1$, so does $c$, which is also not allowed.

Thus, we have $Z \neq 0$. In this case, the vanishing of the first two partials implies $X=Y=Z$. Plugging this into $F_{c}$, we get

$$
0=Z^{4}+Z^{4}+Z^{4}+c Z^{4}+c Z^{4}+c Z^{4}=(1+c) Z^{4} .
$$

This is impossible unless $c=1$ or $Z=0$.

EXAMPLE 3.6. Let $K$ have characteristic 5. Let

$$
P(x)=x^{5}+x^{4}+x^{2}+x+1 .
$$

Then $S=\{z \in K: P(z)=0\}$ contains 5 points and is a unique range set for $\mathcal{A}^{*}(K)$.

Proof. To see that $P$ has distinct roots, we compute

$$
P^{\prime}(x)=-x^{3}+2 x+1=-(x+1)(x+2)^{2},
$$

and we see $P$ and $P^{\prime}$ have no common roots, and hence the roots of $P$ are distinct.

Let $c \neq 0$ be a non-zero constant in $K$, and let

$$
\begin{aligned}
F_{c}(X, Y, Z)= & X^{5}-c Y^{5}+Z\left(X^{4}-c Y^{4}\right)+Z^{3}\left(X^{2}-c Y^{2}\right) \\
& +Z^{4}(X-c Y)+(1-c) Z^{5}
\end{aligned}
$$

which is the projectivization of $P(x)-c P(y)$. As noted before, to check that $S$ is a unique range set, it suffices to check that $F_{c}$ has no rational component when $c \neq 1$ and only has the rational component $X-Y=0$ when $c=1$.

We begin with $F_{1}$. Let

$$
\begin{aligned}
G(X, Y, Z) & =F_{1}(X, Y, Z) /(X-Y) \\
& =(X-Y)^{4}+Z\left(X^{3}+X^{2} Y+X Y^{2}+Y^{3}\right)+Z^{3}(X+Y)+Z^{4} .
\end{aligned}
$$

We compute the three first partial derivatives

$$
\begin{aligned}
& \frac{\partial G}{\partial X}=-(X-Y)^{3}+Z\left(3 X^{2}+2 X Y+Y^{2}\right)+Z^{3} \\
& \frac{\partial G}{\partial Y}=(X-Y)^{3}+Z\left(3 Y^{2}+2 X Y+X^{2}\right)+Z^{3} \\
& \frac{\partial G}{\partial Z}=X^{3}+X^{2} Y+X Y^{2}+Y^{3}+3 Z^{2}(X+Y)-Z^{3} .
\end{aligned}
$$


Singular points occur when all three partials vanish together with $G$. If $Z=0$, the vanishing of $G$ implies $X=Y$. Then the vanishing of $\partial G / \partial Z$ implies $-X^{3}=0$, and so $X=Y=0$, which is not allowed. Thus $Z \neq 0$. In this case, we may assume $Z=1, X=x$, and $Y=y$. Then our equations become

$$
\begin{aligned}
& 0=G=(x-y)^{4}+x^{3}+x^{2} y+x y^{2}+y^{3}+x+y+1, \\
& 0=\frac{\partial G}{\partial X}=-(x-y)^{3}+3 x^{2}+2 x y+y^{2}+1, \\
& 0=\frac{\partial G}{\partial Y}=(x-y)^{3}+3 y^{2}+2 x y+x^{2}+1, \\
& 0=\frac{\partial G}{\partial Z}=x^{3}+x^{2} y+x y^{2}+y^{3}+3 x+3 y-1 .
\end{aligned}
$$

Adding the first two partials, we get

$$
0=-\left(x^{2}+x y+y^{2}\right)+2 \text { or } x^{2}+x y+y^{2}=2 .
$$

Thus,

$$
\begin{aligned}
& x^{3}+x^{2} y+x y^{2}=x\left(x^{2}+x y+y^{2}\right)=2 x, \\
& y^{3}+y^{2} x+y x^{2}=y\left(x^{2}+x y+y^{2}\right)=2 y .
\end{aligned}
$$

Using these in the equation $\partial G / \partial Z=0$, we find

$$
\begin{aligned}
& 0=2 x+y^{3}+3 x+3 y-1=y^{3}-2 y-1, \\
& 0=2 y+x^{3}+3 x+3 y-1=x^{3}-2 x-1 .
\end{aligned}
$$

Plugging into $G=0$, we find

$$
\begin{aligned}
0 & =(x-y)^{4}+x^{3}+x^{2} y+x y^{2}+y^{3}+x+y+1 \\
& =(x-y)^{4}+(1+2 x)+x^{2} y+x y^{2}+(1+2 y)+x+y+1 \\
& =(x-y)^{4}+x^{2} y+x y^{2}-2 x-2 y-2 \\
& =(x-y)^{4}+x\left(x^{2}+x y+y^{2}\right)-x^{3}-2 x-2 y-2 \\
& =(x-y)^{4}+2 x-(1+2 x)-2 x-2 y-2 \\
& =(x-y)^{4}-2(x+y)+2 \\
& =x^{4}+x^{3} y+x^{2} y^{2}+x y^{3}+y^{4}-2(x+y-1) \\
& =(x+y) x^{3}+x^{2} y^{2}+(x+y) y^{3}-2(x+y-1) \\
& =(x+y)(1+2 x)+x^{2} y^{2}+(x+y)(1+2 y)-2(x+y-1) \\
& =2(x+y)^{2}+x^{2} y^{2}+2=2\left(x^{2}+y^{2}\right)+2 .
\end{aligned}
$$

In other words,

$$
x^{2}+y^{2}=-1 \text {. }
$$

Combining (7) and (4), we get $x y=-2$. Plugging in $y=-2 / x$ into (6) and $x=-2 / y$ into $(5)$, we find

$$
x^{3}+x^{2}+2=0 \text { and } y^{3}+y^{2}+2=0 .
$$


Subtracting (5) and (6), we get

$$
x^{2}+2 x-2=0 \text { and } y^{2}+2 y-2=0 .
$$

Adding these equations, we find

$$
x^{2}+y^{2}+2(x+y)+1=0 .
$$

Combining with (7), we see that $y=-x$, and so using (7) once more, we get $x^{2}=y^{2}=2$. Plugging this into (8), we get $x=y=0$, and thus $G=0$ is non-singular and has genus 3 because it has degree 4 .

Now we treat the case $F_{c}$ for $c \neq 0,1$. Again, we compute the three first partials:

$$
\begin{aligned}
& \frac{\partial F_{c}}{\partial X}=-Z X^{3}+2 X Z^{3}+Z^{4}=-Z(X+Z)(X+2 Z)^{2} \\
& \frac{\partial F_{c}}{\partial Y}=c\left(Z Y^{3}-2 Y Z^{3}-Z^{4}\right)=c Z(Y+Z)(Y+2 Z)^{2}, \\
& \frac{\partial F_{c}}{\partial Z}=X^{4}-c Y^{4}+2 Z^{2}\left(c Y^{2}-X^{2}\right)+Z^{3}(c Y-X) .
\end{aligned}
$$

If $Z=0$, then from $\partial F_{c} / \partial Z=0$, we have $X^{4}-c Y^{4}=0$. On the other hand, from $F_{c}=0$, we have $X^{5}-c Y^{5}=0$. Thus, using the same trick as in Example 3.5 (e.g., equation (3)), we see that this is impossible and there are no singular points with $Z=0$. If $Z \neq 0$, we can again assume $Z=1$, and we are working with the following four equations:

$$
\begin{aligned}
& 0=F_{c}=x^{5}-c y^{5}+x^{4}-c y^{4}+x^{2}-c y^{2}+x-c y+1-c, \\
& 0=\frac{\partial F_{c}}{\partial X}=-x^{3}+2 x+1=-(x+1)(x+2)^{2}, \\
& 0=\frac{\partial F_{c}}{\partial Y}=c\left(y^{3}-2 y-1\right)=c(y+1)(y+2)^{2}, \\
& 0=\frac{\partial F_{c}}{\partial Z}=x^{4}-2 x^{2}-x-c\left(y^{4}-2 y^{2}-y\right)=-x \frac{\partial F_{c}}{\partial X}+c y \frac{\partial F_{c}}{\partial Y} .
\end{aligned}
$$

Thus, setting the third partial derivative to zero is redundant. From the vanishing of the first two partials, we see we only have two possibilities for $x$ and two possibilities for $y$. Namely, $x=-1$ or $x=-2$, and $y=-1$ or $y=-2$. Note that $P(-1)=1$ and $P(-2)=2$. From $F_{c}=0$, we get

$$
c=\frac{P(x)}{P(y)},
$$

and so for $F_{c}$ to have singularities, we need $c=1,2$, or $1 / 2=-2$. We have already dealt with the case $c=1$. Now consider the case $c=2$. In that case, the only singular point is $(x, y)=(-2,-1)$. The Taylor expansion about this point is

$$
F_{2}(x, y)=2(x+2)^{2}+(y+1)^{2}+\ldots
$$


Therefore $(-2,-1)$ is an ordinary double point. This means $F_{2}$ must be irreducible, because if it were not irreducible, two components would have to meet at this one singular point in at least multiplicity 4 , and so it could not be an ordinary double point. Thus, $F_{2}$ is irreducible and has genus

$$
\frac{(5-1)(5-2)}{2}-1=5 \text {. }
$$

In the case $F_{-2}$, the singular point is $(-1,-2)$ and the Taylor expansion is

$$
F_{-2}(x, y)=2(x+1)^{2}-(y+2)^{2}+\ldots,
$$

and again the singularity is an ordinary double point, meaning the genus of $F_{-2}$ is again 5 . For all other values of $c$, the genus is 6 , and so we are done.

Notice that in Example 3.5, we were able to find a polynomial $P$ such that the form $F_{c}$ had no singularities, other than those it has to have when $c=1$. Our form $F_{c}$ in Example 3.6 acquires a mild singularity for certain values of $c$. As the degree of the polynomial $P$ increases, it seems difficult to find examples that can be easily worked with where the form $F_{c}$ remains nonsingular for all $c \neq 1$. See [21] and [14] for a further discussion of this aspect of the theory. See also [19] where Voloch studies singularities of plane curves of the form $[P(x)-P(y)] /(x-y)$ in positive characteristic with another application in mind.

4. Proof of Theorem 3.1. The main tool in the proof will be a nonArchimedean positive characteristic analog of Nevanlinna's Second Main Theorem, recently proven by Boutabaa and Escassut [6]. For background on $p$-adic analysis, see [2] and [11], and for a more thorough introduction to nonArchimedean Nevanlinna theory (in characteristic zero), see, for instance, one of the following: [4], [10], [8], [18], [15]. Note that the work of Ru [18] also carries over to positive characteristic without change, but is not sufficient for our application here.

We first introduce some Nevanlinna style notation and then state the Nevanlinna theorem we will apply. As previously, $K$ will be an algebraically closed field complete with respect to a non-Archimedean absolute value of characteristic $p \geq 0$. Let $f$ be a (non-Archimedean) meromorphic function on $K$. For each point $z_{0}$ in $K$ denote by $\omega_{z_{0}}(f)$ the order of vanishing of $f$ at $z_{0}$. That is, if $f\left(z_{0}\right)=0$, then $\omega_{z_{0}}(f)$ denotes the multiplicity of the zero at $z_{0}$. If $f$ has a pole, then $-\omega_{z_{0}}(f)$ denotes the order of the pole. Define

$$
\omega_{z_{0}}^{+}(f)=\max \left\{0, \omega_{z_{0}}(f)\right\} .
$$

For every $r>0$, we define the counting function of zeros by

$$
Z(r, f)=\sum_{0<\left|z_{0}\right|<r} \omega_{z_{0}}^{+}(f) \log \frac{r}{\left|z_{0}\right|}+\omega_{0}^{+}(f) \log r .
$$


If $p>0$, let $u(f)$ be the largest integer such that there exists a meromorphic function $g$ on $K$ such that

$$
f=g^{p^{u(f)}} .
$$

If $p=0$, by convention we let $p^{u(f)}=1$. Next, we define the truncated counting function by

$$
\begin{aligned}
\bar{Z}(r, f)= & \sum_{0<\left|z_{0}\right|<r} \min \left\{1, \omega_{z_{0}}^{+}(f) \bmod p^{u(f)+1}\right\} \log \frac{r}{\left|z_{0}\right|} \\
& +\min \left\{1, \omega_{0}^{+}(f) \bmod p^{u(f)+1}\right\} \log r .
\end{aligned}
$$

In characteristic zero, we simply ignore the $\bmod p^{u(f)+1}$ part. Note that in characteristic 0 , the truncated counting function just counts the zeros of $f$, but ignores their multiplicity. In characteristic $p>0$, it almost does the same thing, but it also ignores zeros which have multiplicity a multiple of $p^{u(f)+1}$. We also consider the counting functions for poles. Namely,

$$
N(r, f)=Z(r, 1 / f) \quad \text { and } \quad \bar{N}(r, f)=\bar{Z}(r, 1 / f) .
$$

Finally, we define an analog of the Nevanlinna characteristic function by

$$
T(r, f)=\max \{Z(r, f), N(r, f)\} .
$$

The theory of valuation (or Newton) polygons easily implies the analog of Nevanlinna's first main theorem:

ThEOREM 4.1 (First Main Theorem). If $f$ is a non-constant meromorphic function on $K$ and $a$ is in $K$, then

$$
T(r, f)=T(r, 1 / f)=T(r, f-a)+O(1) .
$$

Corollary 4.2. If $P$ is a polynomial of degree $d$ and $f$ is a meromorphic function on $K$, then $T(P(f), r)=d T(f, r)+O(1)$.

The analog of the Second Main Theorem that we will need is the following special case of what was proven in [6].

Theorem 4.3 (Second Main Theorem [6]). Let $\alpha_{1}, \ldots, \alpha_{n}$ be $n$ distinct points in $K$ and let $f$ be a meromorphic function on $K$. Then

$$
\frac{n-1}{p^{u(f)}} T(r, f) \leq \sum_{i=1}^{n} \bar{Z}\left(r, f-\alpha_{i}\right)+\bar{N}(r, f)-\log r+O(1)
$$

We will actually only need the theorem in the case $f^{\prime} \not \equiv 0$, which is the case $u(f)=0$. If $f^{\prime} \not \equiv 0$, the standard characteristic zero proofs (e.g., [4], [10]) go through, and this is actually sufficient for our application to unique range sets here.

The following corollary to Theorem 4.3 will also be useful. 
COROLlary 4.4. Let $f$ be an analytic function on $K$ such that $f^{\prime} \not \equiv 0$, and let $\alpha_{1}, \ldots, \alpha_{n}$ be distinct points in $K$. Then

$$
\sum_{j=1}^{n}\left[Z\left(r, f-\alpha_{j}\right)-\bar{Z}\left(r, f-\alpha_{j}\right)\right] \leq T(r, f)-\log r+O(1) .
$$

Proof. From Theorem 4.1,

$$
\sum_{j=1}^{n} Z\left(r, f-\alpha_{j}\right)=n T(r, f)+O(1)
$$

and from Theorem 4.3,

$$
\sum_{j=1}^{n} \bar{Z}\left(r, f-\alpha_{j}\right)-\log r+O(1) \geq(n-1) T(r, f)-\log r+O(1) .
$$

Thus,

$\sum_{j=1}^{n}\left[Z\left(r, f-\alpha_{j}\right)-\bar{Z}\left(r, f-\alpha_{j}\right)\right] \leq n T(r, f)+(1-n) T(r, f)-\log r+O(1)$.

Before going into the proof of Theorem 3.1, we state some helpful propositions.

Proposition 4.5. Let $n, m, a$, and $P$ be as in Theorem 3.1. Let $c \neq 1$. Then either $P-c^{-1}$ or $P-c$ has no multiple roots.

Proof. We assume both $P-c^{-1}$ and $P-c$ have a multiple root and arrive at a contradiction. Note that because $c \neq 1$, zero is not a root of either polynomial. Because $m$ and $n$ are not both divisible by $p$, we can differentiate each polynomial and conclude that because of the double root,

$$
m^{m}(n-m)^{n-m} a^{n}=n^{n}(1-c)^{n-m}=n^{n}\left(1-c^{-1}\right)^{n-m},
$$

and moreover, $n, m$, and $n-m$ are relatively prime to $p$. Hence,

$$
\left(1-c^{-1}\right)^{n-m}=(1-c)^{n-m}
$$

and so $c^{n-m}=(-1)^{n-m}$. However, the assumption that

$$
m^{m}(n-m)^{n-m} a^{n} \neq n^{n}(1-\zeta)^{n-m}
$$

for every $\zeta \in K$ such that $\zeta^{n-m}=(-1)^{n-m}$ excludes the possibility of polynomials of the form $P-\zeta$ having multiple zeros, provided $\zeta \neq 1$. Thus, we conclude that $c=1$, which contradicts our hypothesis.

Proposition 4.6. Let $n>m>1$ be integers satisfying conditions (A1)-(A3) of Theorem 3.1, and let $h$ be a non-constant meromorphic function on $K$. Then there exists an element $\alpha \in K$ such that

$$
\omega_{\alpha}^{+}\left(h^{n}-1\right)>\omega_{\alpha}\left(h^{m}-1\right) .
$$


Proof. Let $n=p^{s} d$ with $d=n|n|_{p} \geq 2$ not dividing $p$. By condition (A3), $d$ does not divide $m$. Therefore, there exists at least one $n$th root of unity $\zeta$ which is not an $m$ th root of unity. If there exists an $\alpha$ such that $h(\alpha)=\zeta$, then we see immediately that this $\alpha$ is such that

$$
\omega_{\alpha}^{+}\left(h^{n}-1\right)>\omega_{\alpha}\left(h^{m}-1\right),
$$

as desired. We may now suppose $h$ omits $\zeta$. On the other hand, since $h$ is meromorphic on $K$, it can omit at most one value in $K \cup\{\infty\}$ (by for example Theorem 4.3). Thus, if $h$ omits $\zeta$ it takes on every other value.

Case $s>0$. Because $|(n, m)|_{p}=1$, we have $(m, p)=1$, and hence $x^{m}-1$ has $m$ distinct zeros. On the other hand, 1 is a zero of order $p^{s}$ for $x^{n}-1$. Let $\alpha$ be a point such that $h(\alpha)=1$. Then

$$
\omega_{\alpha}\left(h^{n}-1\right)=p^{s} \omega_{\alpha}\left(h^{m}-1\right),
$$

as desired.

Case $s=0$. In this case the group of $n$th roots of unity has $n \geq 3$ distinct elements. The proper subgroup of elements which are both $m$ th and $n$th roots of unity has order dividing $n$, and hence, in addition to $\zeta$, there is at least one other root $\eta$ such that $\eta^{n}=1$, but $\eta^{m} \neq 1$. Letting $\alpha$ be such that $h(\alpha)=\eta$ completes the proof of the proposition.

The following proposition shows us that we only need to consider functions $f$ and $g$ such that $f^{\prime}$ and $g^{\prime}$ are not identically zero.

Proposition 4.7. Let $K, p, a, P, n$, and $m$ be as in Theorem 3.1. Let $f$ and $g$ be in $\mathcal{A}^{*}(K)$ such that $P(f)=c P(g)$. Then there exist functions $f_{s}$ and $g_{s}$ in $\mathcal{A}^{*}(K)$ and elements $a_{s} \neq 0$ and $c_{s} \neq 0$ in $K$ such that if

$$
P_{s}(x)=x^{n}-a_{s} x^{m}+1,
$$

then

$$
P_{s}\left(f_{s}\right)=c_{s} P\left(g_{s}\right) \text {. }
$$

Moreover, $a_{s}$ satisfies all the same conditions as a stated in Theorem 3.1, and $f_{s}^{\prime} \not \equiv 0$ and $g_{s}^{\prime} \not \equiv 0$.

Proof. By differentiating $P(f)=c P(g)$, we find

$$
P^{\prime}(f) f^{\prime}=c P^{\prime}(g) g^{\prime}
$$

By either condition (A2) or (B2), we have $|(n, m)|_{p}=1$, so $P^{\prime}$ is not identically zero. Thus, either $f^{\prime}$ and $g^{\prime}$ are identically zero or both are not identically zero. If they are both not identically zero, we leave everything alone and we are done.

Otherwise, we can find functions $f_{1}$ and $g_{1}$ in $\mathcal{A}^{*}(K)$ such that $f_{1}^{p}=f$ and $g_{1}^{p}=g$. Then consider

$$
P_{1}(x)=x^{n}-a_{1} x+1,
$$


where $a_{1}$ is chosen such that $a_{1}^{p}=a$. Then

$$
\left[P_{1}\left(f_{1}\right)\right]^{p}=P(f)=c P(g)=\left[c_{1} P_{1}\left(g_{1}\right)\right]^{p},
$$

where $c_{1}^{p}=c$. Replacing $f$ by $f_{1}, g$ by $g_{1}$, and $P$ by $P_{1}$ we continue by induction until both derivatives do not vanish. Continuing this process inductively a finite number of times we will arrive at functions $f_{s}$ and $g_{s}$ whose derivatives do not vanish identically.

It remains to check that

$$
m^{m}(n-m)^{n-m} a_{s}^{n} \neq n^{n}
$$

and

$$
m^{m}(n-m)^{n-m} a_{s}^{n} \neq n^{n}(1-\zeta)^{p^{d}(n-m)}
$$

for all positive $d$. But, if we had equality somewhere here, then we could raise both sides to the $p^{s}$ power (which fixes the integer terms) and contradict our hypothesis on $a=a_{s}^{p^{s}}$.

Proof of Theorem 3.1. The conditions on a make it clear that $P$ has distinct roots. They also ensure that $P-\zeta$ has distinct roots for all $\zeta \neq 1$ such that $\zeta^{n-m}=(-1)^{n-m}$.

If $f$ and $g$ are in $\mathcal{A}^{*}(K)$ and share $S$, then $P(f)=c P(g)$ for some constant $c$, by Proposition 2.1.

If we are in positive characteristic, we can, thanks to Proposition 4.7, assume, without loss of generality, that $f^{\prime} g^{\prime} \not \equiv 0$.

We will use $P(f)=c P(g)$ to create a non-Archimedean meromorphic function which takes on too many values with high ramification, contradicting Theorem 4.3.

We now show $c=1$. Suppose $c \neq 1$ and let

$$
Q(x)=x^{n}-a x^{m}+1-1 / c .
$$

Then

$$
Q(g)=P(g)-c^{-1}=c^{-1}(c P(g)-1)=c^{-1}(P(f)-1)=c^{-1}\left(f^{n}-a f^{m}\right) .
$$

Similarly, let $Q_{1}(x)=P(x)-c$. Then

$$
Q_{1}(f)=P(f)-c=c\left(c^{-1} P(f)-1\right)=c(P(g)-1)=c\left(g^{n}-a g^{m}\right) .
$$

By Proposition 4.5, at least one of the two polynomials $Q$ of $Q_{1}$ has distinct zeros. We may assume $Q$ has distinct zeros by exchanging $f$ and $g$ if necessary. Thus, write

$$
Q(x)=\prod_{j=1}^{n}\left(x-e_{j}\right),
$$


with the $e_{j}$ distinct. Then

$$
f^{n}-a f^{m}=\prod_{j=1}^{n}\left(f-e_{j}\right) .
$$

This is the important point, because then

$$
\begin{aligned}
Z(r, Q(g))-\bar{Z}(r, Q(g)) & =Z\left(r, f^{n}-a f^{m}\right)-\bar{Z}\left(r, f^{n}-a f^{m}\right) \\
& \geq(m-1) Z(r, f) .
\end{aligned}
$$

On the other hand, using Corollary 4.4, we have

$$
\begin{aligned}
Z(r, Q(g))-\bar{Z}(r, Q(g)) & =\sum_{j=1}^{n}\left[Z\left(r, g-e_{j}\right)-\bar{Z}\left(r, g-e_{j}\right)\right] \\
& \leq T(r, g)-\log r+O(1) .
\end{aligned}
$$

But, by Corollary 4.2 ,

$$
n T(r, f)=T(r, P(f))+O(1)=T(r, P(g))+O(1)=n T(r, g)+O(1),
$$

and hence

$$
T(r, g)=T(r, f)+O(1) .
$$

Hence, combining (9) and (10), we get

$$
(m-1) T(r, f)=(m-1) Z(r, f) \leq T(r, g)-\log r+O(1),
$$

and hence $m<1$, contradicting our hypothesis. Thus, $c=1$.

Now, let $h=f / g$. Because $P(f)=P(g)$, we have

$$
g^{n-m}=a \frac{h^{m}-1}{h^{n}-1} .
$$

The remainder of the proof consists of showing $h$ is constant and therefore so are $f$ and $g$, which contradicts the hypothesis of the theorem. The proof splits into two cases, depending on whether conditions (A1)-(A3) are satisfied or conditions (B1)-(B2) are satisfied.

Case $m$ and $n$ satisfy (A1)-(A3). If $h$ is not constant, then Proposition 4.6 says there exists an element $\alpha$ in $K$ such that

$$
\omega_{\alpha}^{+}\left(h^{n}-1\right)>\omega_{\alpha}\left(h^{m}-1\right) .
$$

Thus, $\alpha$ is a pole of $g^{n-m}$, which is a contradiction. Hence $h$ is constant, and therefore so are $f$ and $g$.

Case $m$ and $n$ satisfy (B1)-(B2). In this case we assume $(n, m)=1$, and so there are no non-trivial $n$th roots of unity which are also $m$ th roots of unity. Since at least one of $n$ or $m$ must be prime to $p$, there are at least $m-1$ elements $\zeta_{1}, \ldots, \zeta_{m-1}$ of $K$ which are either $n$th or $m$ th roots of 1 , but not both. Suppose there exists a point $\alpha$ in $K$ such that $h(\alpha)=\zeta_{j}$. Then

$$
g^{n-m}(\alpha)=a \frac{h^{m}(\alpha)-1}{h^{n}(\alpha)-1}=0 .
$$


Note that the possibility of $\infty$ is excluded since $g$ is analytic. Thus, $\alpha$ is a zero of $h$ of order at least $n-m$. Hence, we conclude from Theorem 4.3 that

$$
\begin{aligned}
(m-2) T(r, h) & \leq \sum_{j=1}^{m-1} \bar{Z}\left(r, h-\zeta_{j}\right)+N(r, h)-\log r+O(1) \\
& \leq(n-m) \sum_{j=1}^{m-1} \bar{Z}\left(r, h-\zeta_{j}\right)+N(r, h)-\log r+O(1) .
\end{aligned}
$$

Now using Theorem 4.1, we conclude

$$
(m-2) T(r, h) \leq\left(\frac{m-1}{n-m}+1\right) T(r, h)-\log r+O(1) \text {. }
$$

Hence,

$$
m-2<\frac{m-1}{n-m}+1 .
$$

We have assumed $n-m \geq 2$, so we conclude $m<5$, which contradicts condition (B1).

\section{References}

[1] W. W. Adams and E. G. Straus, Non-Archimedean analytic functions taking the same values at the same points, Illinois J. Math. 15 (1971), 418-424.

[2] Y. Amice, Les nombres p-adiques, Presses Univ. France, 1975.

[3] V. Berkovich, Spectral Theory and Analytic Geometry over Non-Archimedean Fields, Math. Surveys Monographs 33, Amer. Math. Soc., 1990.

[4] A. Boutabaa, Théorie de Nevanlinna p-adique, Manuscripta Math. 67 (1990), 251269.

[5] A. Boutabaa and A. Escassut, Property $f^{-1}(S)=g^{-1}(S)$ for $p$-adic entire and meromorphic functions, Rend. Circ. Mat. Palermo (2) 49 (2000), 501-520.

[6] -, 一, Nevanlinna theory in characteristic $p$ and applications, preprint.

[7] A. Boutabaa, A. Escassut and L. Haddad, On uniqueness of $p$-adic entire functions, Indag. Math. 8 (1997), 145-155.

[8] W. Cherry, A survey of Nevanlinna theory over non-Archimedean fields, Bull. Hong Kong Math. Soc. 1 (1997), 235-249.

[9] W. Cherry and C.-C. Yang, Uniqueness of non-Archimedean entire functions sharing sets of values counting multiplicity, Proc. Amer. Math. Soc. 127 (1999), 967-971.

[10] W. Cherry and Z. Ye, Non-Archimedean Nevanlinna theory in several variables and the non-Archimedean Nevanlinna inverse problem, Trans. Amer. Math. Soc. 349 (1997), 5043-5071.

[11] A. Escassut, Analytic Elements in p-adic Analysis, World Sci., 1995.

[12] W. Fulton, Algebraic Curves. An Introduction to Algebraic Geometry, Benjamin, 1969.

[13] F. Gross, Factorization of meromorphic functions and some open problems, in: Complex Analysis (Lexington, KY, 1976), Lecture Notes in Math. 599, Springer, 1977, $51-67$. 
[14] Ha Huy Khoai and Ta Thi Hoai An, On uniqueness polynomials and bi-URS for p-adic meromorphic functions, J. Number Theory 87 (2001), 211-221.

[15] P.-C. Hu and C.-C. Yang, Meromorphic Functions over Non-Archimedean Fields, Math. Appl. 522, Kluwer, 2000.

[16] L.-W. Liao and C.-C. Yang, On the cardinality of the unique range sets for meromorphic and entire functions, Indian J. Pure Appl. Math. 31 (2000), 431-440.

[17] I. V. Ostrovskii, F. B. Pakovitch, and M. G. Zaidenberg, A remark on complex polynomials of least deviation, Internat. Math. Res. Notices 1996, 699-703.

[18] M. Ru, A note on p-adic Nevanlinna theory, Proc. Amer. Math. Soc. 129 (2001), 1263-1269.

[19] J. F. Voloch, On the number of values taken by a polynomial over a finite field, Acta Arith. 52 (1989), 197-201.

[20] - private communication, April 7, 1997.

[21] J. T.-Y. Wang, Uniqueness polynomials and bi-unique range sets for rational functions and non-Archimedean meromorphic functions, Acta Arith., to appear.

Laboratoire de Mathématiques Pures

Université Blaise Pascal (Clermont-Ferrand)

Les Cézeaux

63177 Aubière Cedex, France

E-mail: boutabaa@math.univ-bpclermont.fr escassut@math.univ-bpclermont.fr
Department of Mathematics University of North Texas Denton, TX 76203, U.S.A. E-mail: wcherry@unt.edu

Received on 16.7.2001

and in revised form on 26.9.2001 